\title{
Molecular cloning and characterization of the MsHSP17.7 gene from Medicago sativa $\mathrm{L}$.
}

\author{
Zhen-yi Li $^{1} \cdot$ Rui-cai Long ${ }^{1}$ Tie-jun Zhang ${ }^{1} \cdot$ Qing-chuan Yang $^{1} \cdot$ \\ Jun-mei Kang ${ }^{1}$
}

Received: 1 September 2015/Accepted: 12 May 2016/Published online: 19 May 2016

(c) The Author(s) 2016. This article is published with open access at Springerlink.com

\begin{abstract}
Heat shock proteins (HSPs) are ubiquitous protective proteins that play crucial roles in plant development and adaptation to stress, and the aim of this study is to characterize the $H S P$ gene in alfalfa. Here we isolated a small heat shock protein gene (MSHSP17.7) from alfalfa by homology-based cloning. MsHSP17.7 contains a 477-bp open reading frame and encodes a protein of $17.70-\mathrm{kDa}$. The amino acid sequence shares high identity with MtHSP (93.98 \%), PsHSP17.1 (83.13\%), GmHSP17.9 (74.10 \%) and SIHSP17.6 (79.25\%). Phylogenetic analysis revealed that MsHSP17.7 belongs to the group of cytosolic class II small heat shock proteins (sHSP), and likely localizes to the cytoplasm. Quantitative RT-PCR indicated that MsHSP17.7 was induced by heat shock, high salinity, peroxide and drought stress. Prokaryotic expression indicated that the salt and peroxide tolerance of Escherichia coli was remarkably enhanced. Transgenic Arabidopsis plants overexpressing MsHSP17.7 exhibited increased root length of transgenic Arabidopsis lines under salt stress compared to the wild-type line. The malondialdehyde (MDA) levels in the transgenic lines were significantly lower than in wild-type, although proline levels were similar between transgenic and wild-type lines. MsHSP17.7 was induced by heat shock, high salinity, oxidative stress and drought stress. Overexpression
\end{abstract}

Electronic supplementary material The online version of this article (doi:10.1007/s11033-016-4008-9) contains supplementary material, which is available to authorized users.

Jun-mei Kang

kangjmei@126.com

1 Institute of Animal Sciences, Chinese Academy of Agricultural Sciences, Beijing 100193,

People's Republic of China analysis suggests that MsHSP17.7 might play a key role in response to high salinity stress.

Keywords Heat shock protein - Homology cloning · Medicago sativa $\cdot$ RT-qPCR $\cdot$ Salt stress

\section{Introduction}

Plant HSPs normally participate in responses to drought, heat shock, salinity, heavy metals and peroxide stress [1]. HSPs act as molecular chaperones that bind other proteins to maintain steady-state target protein and promote the recovery of denatured proteins, which allows homeostasis of the internal environment during plant development and stress adaptation [2]. Small heat shock proteins (sHSPs, 15-42 kDa) form 200-800 kDa multimeric chaperone complexes [3] and are divided into six classes based on sequence similarity and cellular location. Class CI, CII and CIII sHSPs localize to the cytosol or nucleus [4], as well as the endoplasmic reticulum, mitochondria, and membranes [5]. sHSPs just bind to misfolded or denatured protein substrates, whereas refolding steps are mediated by Hsp70/ Hsp100 complexes [6], preventing irreversible unfolding or aberrant protein aggregation [7]. Their ability to prevent irreversible protein aggregation and to resolubilize aggregated proteins allows native proteins to remain in a stable state. Thus, sHSPs have been described as the 'paramedics of the cell' $[8,9]$.

Several studies have shown that numerous plant sHSPs participate in the development of nutritive tissue, embryogenesis, germination and fruit production [10]. In addition, sHSP plays a significant role in the response to abiotic stresses. The Arabidopsis AtHsp15.7 gene is expressed at very low levels in a normal environment but is 
highly expressed upon heat shock or oxidative stress [11]. A previous study demonstrated that overexpression of sHSP17.7 increased drought tolerance in transgenic rice seedlings [7], and Lee et al. found that overexpression of the MsHSP23 gene enhanced salinity and arsenic tolerance in transgenic tobacco [3].

Alfalfa (Medicago sativa L.) is an important leguminous forage plant and is cultivated worldwide because of its high nutritional value and yield. Adverse external conditions, such as salinity, drought, high temperature and other types of stress, have a negative impact on the yield and quality of alfalfa. Therefore, improved stress resistance is a priority in breeding efforts to produce novel varieties of alfalfa that are better adapted to specific local environments and varying climate conditions. Here, we report the characterization and function of MsHSP17.7 gene from alfalfa, a possible candidate gene for stress tolerance in $M$. sativa.

\section{Materials and methods}

\section{Plant materials and growth conditions}

M. sativa L. cv. Zhongmu No.1 and Arabidopsis thaliana (ecotype; Col-0) were used for gene cloning and genetic modifications. Thirty alfalfa seedlings per pot were cultured in a Hoagland hydroculture system in a plant growth chamber with $60 \%$ humidity, a temperature of $24^{\circ} \mathrm{C}$, and

\section{The photographs and figures in the text}

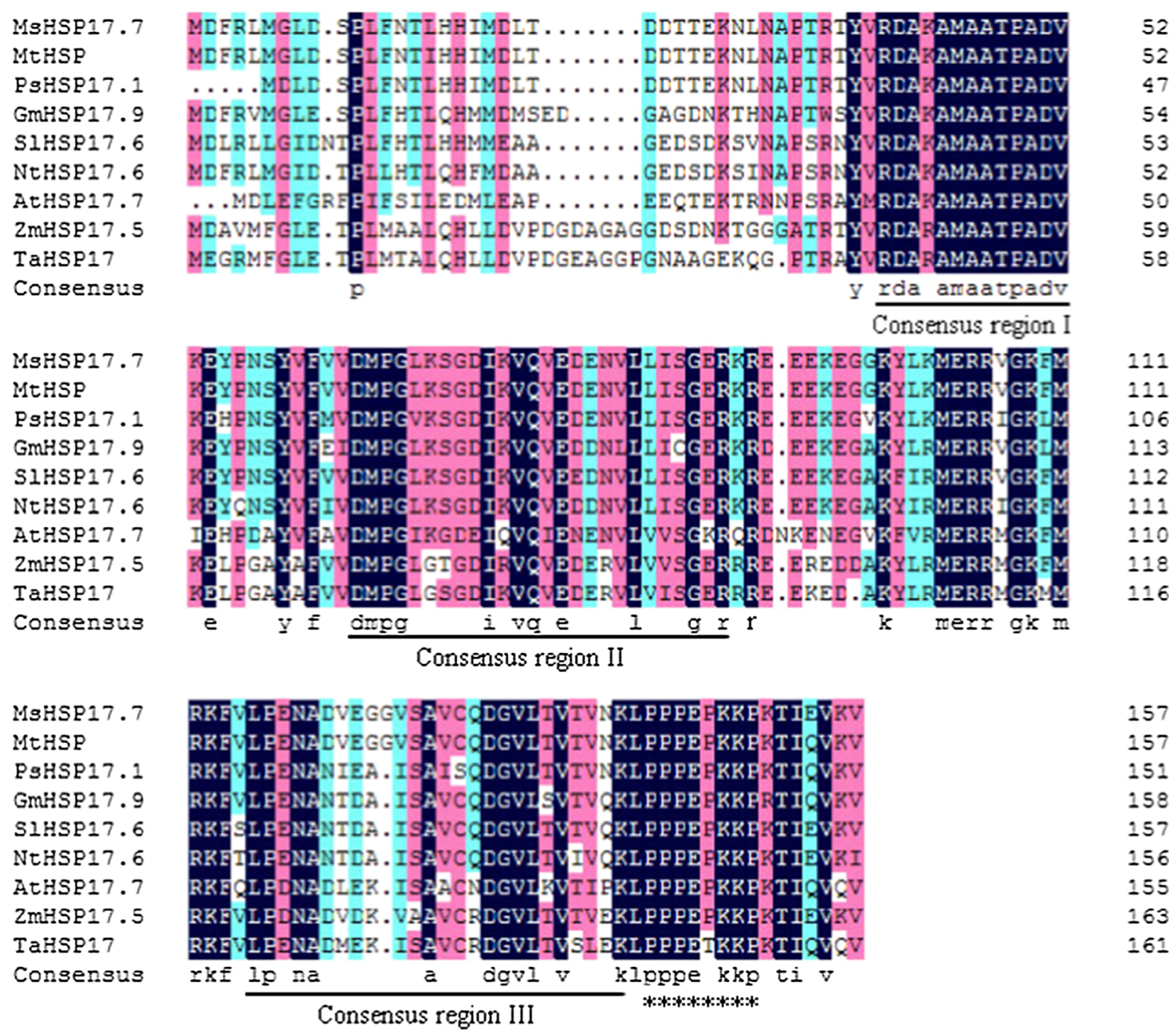

Fig. 1 Multiple sequence alignment of MsHSP17.7 with other plant sHSPs. Black indicates that $100 \%$ of the nine sequences have the same amino acid at a given position, pink indicates that $75 \%$ of the sequences are conserved, and blue indicates that $50 \%$ of the sequences are conserved. Conserved motifs are underlined. Asterisk indicates a polyproline motif. The accession numbers of the sHSPs and its similarity are as follows, MsHSP17.7 (Medicago sativa,
A0A060CW40); MtHSP (Medicago truncatula, G7J8C7), $93.98 \%$; PsHSP17.1 (Pisum sativum, P19242), $83.13 \%$; GmHSP17.9 (Glycine max, P05477), 74.10\%; SIHSP17.6 (Solanum lycopersicum, Q96489), 79.25\%; Nthsp17.6 (Nicotiana tabacum, A0A077DBK4), $74.70 \%$; AtHAP17.7 (Arabidopsis thaliana, O81822), $56.63 \%$; ZmHSP17.5 (Zea mays, B6SJE9), $65.66 \%$; TaHSP17 (Triticum aestivum, A0A077RX64), $63.25 \%$. (Color figure online) 
a $16 \mathrm{~h}$ light $/ 8 \mathrm{~h}$ dark cycle. Wild-type $A$. thaliana was cultured in pots containing a vermiculite/soil (1:3) mixture under the conditions described above.

\section{Cloning of the MsHSP17.7 gene and bioinformatics analysis}

Homology-based cloning was performed to obtain the open reading frame (ORF) of MsHSP17.7. Plant total RNA was extracted from alfalfa using the MiniBEST plant RNA extraction kit (Takara Biotech Co., Ltd., Dalian, China), and cDNA was subsequently obtained using the PrimeScript RT-PCR Kit (Takara). MsHSP-F (sequence: 5'CCTCCCATAATCTTCCAACCAC- $3^{\prime}$ ) was used as the sense primer, and MsHSP-R (sequence: 5'-CAAAAAAC CATTGCCACACACG- $3^{\prime}$ ) was used as the antisense primer. DNA fragment were cloned using alfalfa cDNA as the template by ordinary PCR. The obtained DNA fragment was then ligated into the pEASY-T1 vector, which was purified from positive Escherichia coli colonies containing the MsHSP17.7 gene and the insert was sequenced.

We analyzed the sequence using the abc website (http:// abc.cbi.pku.edu.cn/). The ORF was found by plotorf ([v 6.0.1]; Alan Bleasby, European Bioinformatics Institute, UK) and translated into an amino acid sequence. The following analyses were performed: protein hydrophobicity (Protscale, http://web.expasy.org/protscale/), signal prediction (SignalP 4.1, http://www.cbs.dtu.dk/services/Sig nalP/), transmembrane motif prediction (TMHMM, http:// www.cbs.dtu.dk/services/TMHMM-2.0/), protein secondary structure analysis (Garnier [v6.0.1]; William Pearson, European Bioinformatics Institute, UK), subcellular location prediction (ProtComp, http://www.softberry.com) and multiple sequence alignment (DNAMAN 6.0; Lynnon Biosoft, USA). The phylogenetic tree of MsHSP17.7 was constructed using MEGA5.1 [12] software.

\section{Subcellular localization of the MsHSP17.7}

The coding sequence of MsHSP17.7 was amplified from plasmid pEASY-T1 using the forward primer pA7-F ( $5^{\prime}-$ CCGCTCGAGATGGATTTCAGGCTAATGGGT-3'; the XhoI site is underlined) and reverse primer pA7-R $\left(5^{\prime}\right.$ CGGACTAGTAGCAACCTTAACCTCAATAGT-3'; the SpeI site is underlined). Then, the fragment was digested with $\mathrm{XhoI}$ and SpeI and ligated into the similarly digested vector pA7-GFP (Supplementary Fig. 4) which contains the CaMV 35S-promoter and the green fluorescent protein (GFP) gene. pA7-GFP and MsHSP17.7-GFP gene fusion plasmids were transformed into onion epidermal cells using a particle gun (PDS1000/He; Bio-Rad, USA), and the bombarded tissues were incubated on a fresh plate for $16 \mathrm{~h}$ in dark environment. Then the bombarded onion epidermal cells were placed in $200 \mathrm{mM} \mathrm{NaCl}$ for 5-10 min for plasmolysis. Cells were visualized with a confocal laserscanning microscope (TE2000-E; Nikon, Japan).

\section{Expression analysis of MsHSP17.7}

To investigate the expression pattern of MsHSP17.7 in alfalfa under heat shock, high salinity, oxidative stress and drought stress, 25-day-old alfalfa seedlings were treated over a 24 -h period to induce heat shock $\left(37^{\circ} \mathrm{C}\right)$, salt stress (200 mM NaCl), oxidative injury $\left(15 \mathrm{mM} \mathrm{H}_{2} \mathrm{O}_{2}\right)$ or drought conditions (200 g 1 ${ }^{-1}$ polyethylene glycol (PEG) 6000) in Hoagland solution. Three alfalfa seedlings were randomly selected at $0,2,4,8,12$ and $24 \mathrm{~h}$, and total RNA was extracted from roots, stems and leaves. Subsquently cDNA was reversely transcribed using the PrimeScript RT-PCR Kit (Takara) described above. Then quantitative reverse-transcription PCR (RT-qPCR) were performed on roots, stems and leaves of alfalfa. The specific primers $M s H S P 17.7 \mathrm{qhsp}-\mathrm{f}$ (5'-CACCACATAATGGACCTCACAGAT-3') and qhsp-r (5'-TGATGTCACCTGATTTCAACCCTG-3') were used in assays. The alfalfa $\beta$-actin gene (GenBank: JQ028730.1) was used as an internal control with the primers qact-f $\left(5^{\prime}-\right.$ CAAAAGATGGCAGATGCTGAGGAT- $\left.3^{\prime}\right)$ and qact-r $\left(5^{\prime}\right.$ CATGACACCAGTATGACGAGGTCG-3'). Then quantitative reverse transcription PCR (RT-qPCR) reactions were performed on roots, stems and leaves. The RT-qPCR protocol was performed as specified by the manufacturer's instructions for the SYBR Primix Ex Tap II kit (Takara). The mean threshold cycle $(\mathrm{Ct})$ was used as a reference value to calculate the level of each mRNA. Three biological replicates and per replicate contains three alfalfa seedlings were performed. The variance was subjected to a least significant difference (LSD) test using SAS software (version 9.13).

\section{Expression of MsHSP17.7 in E. coli and salinity and oxidative stress survival assays}

The complete MsHSP17.7 ORF was amplified with forward primer EHSP-F (5'-ATGGATTTCAGGCTAATGGGTTT GG-3 $\left.{ }^{\prime}\right)$ and reverse primer EHSP-R (5'-TCAAGCAACCT TAACCTCAATAGTCT- $3^{\prime}$ ) and ligated into a prokaryotic expression vector (pEASY-E2, Novagen, USA) to generate the expression plasmid pEASY-E2/MsHSP17.7. Then, the plasmid was transformed into the E. coli strain Transetta DE3, which was cultured at $37{ }^{\circ} \mathrm{C}$ with shaking at $200 \mathrm{rpm}$. During $\log$ phase, $1 \mathrm{mM}$ IPTG was added to bacterium solution to induce protein expression. The bacterial suspension was harvested by centrifugation for $0,3,5,7$ and $9 \mathrm{~h}$, and the fusion protein was separated by $12 \%$ SDS-PAGE and Coomassie brilliant blue (CBB) staining.

Tolerance to salt and oxidative stresses were tested by growing bacteria in LB medium supplemented with 


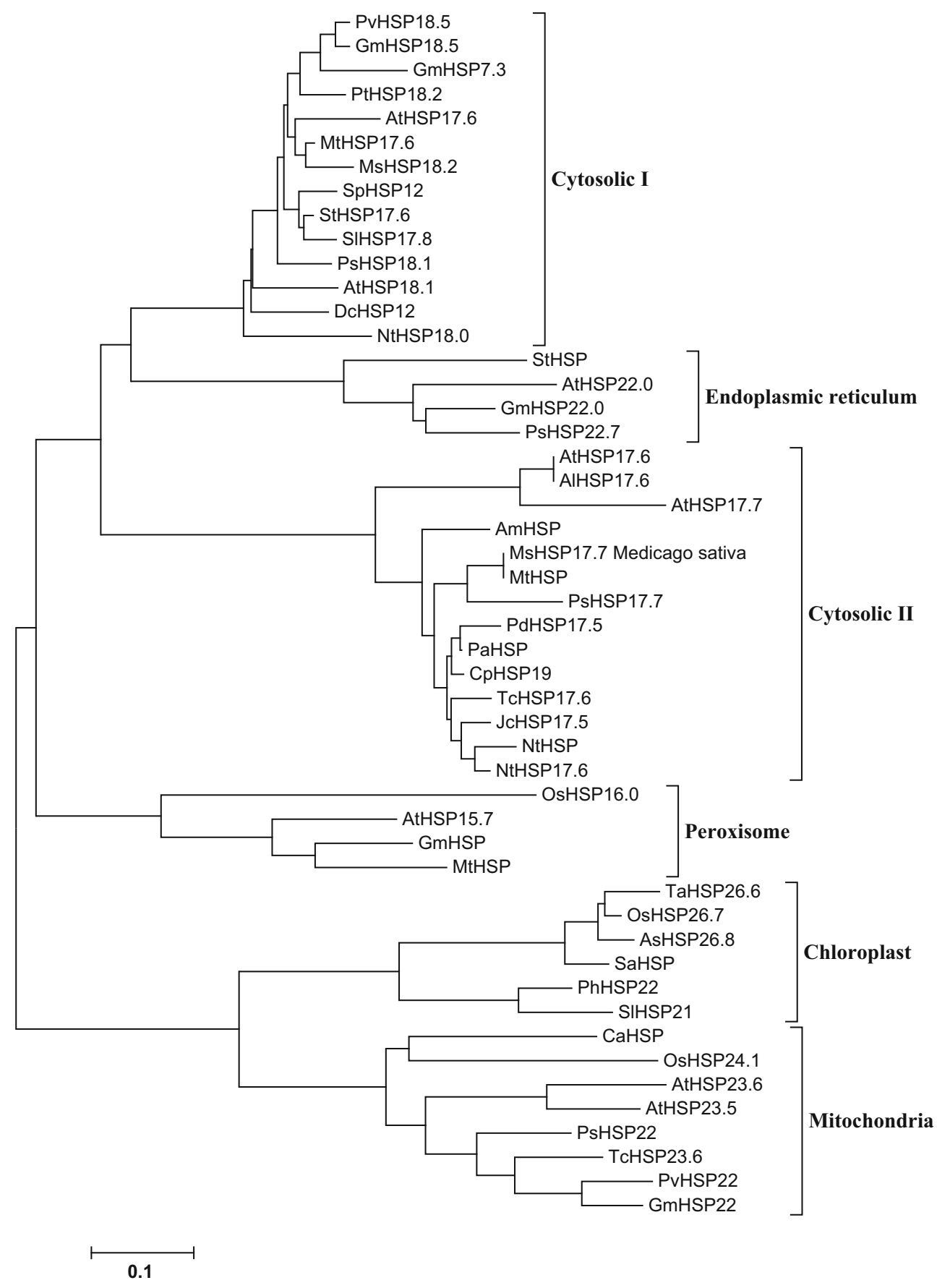


4Fig. 2 Neighbor-joining phylogenetic tree of MsHSP17.7 and sHSPs from other plant species. The phylogenetic tree was constructed based on similarities with 50 plant sHSPs, which divided the sHSP gene family into six clades. Amino acid sequences used in the analysis were retrieved from GenBank or EMBL. Their database accession numbers are as follows: AtHSP18.1 (Arabidopsis thaliana, P19037); NtHSP18.0 (Nicotiana tabacum, A0A068LKK5); PvHSP18.5 (Phaseolus vulgaris, T2DN13); DcHSP18.0 (Daucus carota, P27397); GmHSP18.5 (Glycine max, P05478); GmHSP17.3 (Glycine max, P02519); PtHSP18.2 (Populus trichocarpa, B9HHJ3); PsHSP18.1 (Pisum sativum, P19243); AtHSP17.6 (Arabidopsis thaliana, Q9ZW31); MtHSP17.6 (Medicago truncatula, Q2HTU2); MsHSP18.2 (Medicago sativa, P27880); StHSP17.6 (Solanum tuberosum, W5XNJ3); S1HSP17.8 (Solanum lycopersicum, P30221); SpHSP17.6 (Solanum peruvianum, O82012); StHSP (Solanum tuberosum, Q41218); AtHSP22.0 (Arabidopsis thaliana, Q38806); GmHSP22.0 (Glycine max, P30236); PsHSP 22.7 (Pisum sativum, P19244); PdHSP17.5 (Prunus dulcis, Q9XGS6); PIHSP (Prunus salicina, C9EIM5); CpHSP19 (Citrus paradisi, Q84LP5); TcHSP17.6 (Theobroma cacao, A0A061GJC5); JcHSP17.5 (Jatropha curcas, D5JG84); NtHSP (Nicotiana tabacum, Q53E18); NtHSP17.6 (Arabidopsis thaliana, A0A077DBK4); AmHSP (Ammopiptanthus mongolicus, S5TJ94); PsHSP17.1 (Pisum sativum, P19242); MtHSP (Medicago truncatula, G7J8C7); AlHSP17.7 (Arabidopsis thaliana, O81822); AtHSP17.6 (Arabidopsis thaliana, P29830); AtHSP17.6 (Arabidopsis thaliana, P29830); OsHSP16.0 (Oryza sativa, Q652V8); AtHSP15.7 (Arabidopsis thaliana, Q9FHQ3); GmHSP (Glycine max, B0M1A7); MtHSP (Medicago truncatula, G7KG40); TaHSP26.6 (Triticum aestivum, Q9SBB7); OsHSP26.7 (Oryza sativa, Q10P60); AsHSP26.8 (Agrostis stolonifera, Q8GV37); SaHSP (Spartina alterniflora, J7H8N1); PhHSP22 (Petunia hybrida, P30222); SIHSP21 (Solanum lycopersicum, Q95661); CaHSP (Capsicum aпnиит, D9IAX1); OsHSP24.1 (Oryza sativa, Q6Z7V2); AtHSP23.6 (Arabidopsis thaliana, Q96331); AtHSP23.5 (Arabidopsis thaliana, Q9FGM9); PsHSP22 M (Pisum sativum, P46254); TcHSP23.6 (Theobroma cacao, A0A061FZB7); PvHSP22 (Phaseolus vulgaris, V5N8V1); GmHSP22 (Glycine max, Q39818)

$100 \mathrm{mM} \mathrm{NaCl}$ and $15 \mathrm{mM} \mathrm{H}_{2} \mathrm{O}_{2}$. Then, E. coli cells expressing the pEASY-E2/MsHSP17.7 plasmid were incubated at $37{ }^{\circ} \mathrm{C}$ with shaking at $180 \mathrm{rpm}$. Empty vectortransfected $E$. coli was used as the control, and the growth rate was determined by measuring the OD600 value at 0,2 , $4,6,8,10,12$ and $24 \mathrm{~h}$ [3].

\section{Construction of the plant expression vector and generation of transgenic Arabidopsis}

MsHSP17.7 cDNA containing XbaI and BamHI restriction sites was cloned with the primers pBI-F (5'-TGCTCTA GAATGGATTTCAGGCTAATGGGT- ${ }^{\prime}, \quad X b a \mathrm{I}$ site is underline) and pBI-R (5'-CGGGATCCAGCAACCTTA ACCTCAATAGTC-3', BamHI site underline). The XbaIBamHI fragment was inserted into pBI121 encoding the CaMV 35S promoter. Subsequently, the pBI121-35SMsHSP17.7 recombinant vector was transformed into Agrobacterium GV3101 using the freeze-thaw method. Then, Agrobacterium-mediated transformation of Arabidopsis was performed with the floral dip method [13]. The seeds obtained were screened on $1 / 2 \mathrm{MS}$ medium with $50 \mathrm{mg} \mathrm{l}^{-1}$ kanamycin.
The transformed Arabidopsis seedlings were transplanted into pots under the conditions described above.

To identify transgenic $A$. thaliana, genomic PCR and RTPCR analyses were performed comparing wild-type and transgenic lines. The primers pBI-F and pBI-R were used to amplify the MsHSP17.7 gene using the genomic gene in $A$. thaliana plants as a template for PCR. Then, At-act-F ( $5^{\prime}$ GAAGTCTTGTTCCAGCCCTCGTTTG-3') and At-act-R (5'-GAACCACCGATCCAGACACTGTACT-3') were used to amplify the A. thaliana actin 2 gene (GenBank: NM_112764.3) based the cDNA template as a control. Additionally, pBI-F and pBI-R were used to amplify MsHSP17.7 from the cDNA template. In this experiment, T3 transgenic Arabidopsis homozygous lines, T31 and T37, were randomly selected and used throughout the study.

To identify the $M s H S P 17.7$ was integrated into the position of the genome in A. thaliana, thermal asymmetric interlaced PCR (TAIL-PCR) was performed in this assay. The primers and cycling conditions of experiment protocol was described as Liu et al.'s paper [14]. Specific primer TR1 (5'-TGCAT GACGTTATTTATGAGATGGGTT-3') or TL1 (5'-TAGG GTTCCTATAGGGTTTCGCTCA-3') was used in primary reaction, specific primer TR2 (5'-TATGATTAGAGTCCCG CAATTATACA-3') or TL2 (5'-GTGTTGAGCATATAAG AAACCCTTAG- $3^{\prime}$ ) was used in secondary reaction, and TR3 (5'-CTAGGATAAATTATCGC-3') or TL3 (5'-CCTAAAA CCAAAATCCAG- $3^{\prime}$ ) was used in tertiary reaction [14].

\section{Analysis of transgenic Arabidopsis under stress conditions}

For salt stress treatment, 15 seeds were randomly taken from T3 transgenic lines and wild-type A. thaliana. Arabidopsis were germinated on 1/2 MS medium containing $150 \mathrm{mM} \mathrm{NaCl}$ for 12 days, and the lengths of their roots were measured. As a control, 4-week wild-type Arabidopsis, then $\mathrm{NaCl}$ was added to the Hoagland solution to obtain a final concentration of $200 \mathrm{mM} \mathrm{NaCl}$. At $36 \mathrm{~h}$, the MDA and proline contents were measured. The MDA content was determined using the thiobarbituric acid (TBA) reaction, as described by Heath and Packer (1968). Three technical replicates and per replicate contains three Arabidopsis were performed. The variance was subjected to a LSD test using SAS software (version 9.13).

\section{Results}

\section{Cloning and molecular characterization of $\mathrm{MsHSP17.7}$}

A 686 bp cDNA fragment containing a 477 bp ORF was amplified from alfalfa by homology cloning and designated 


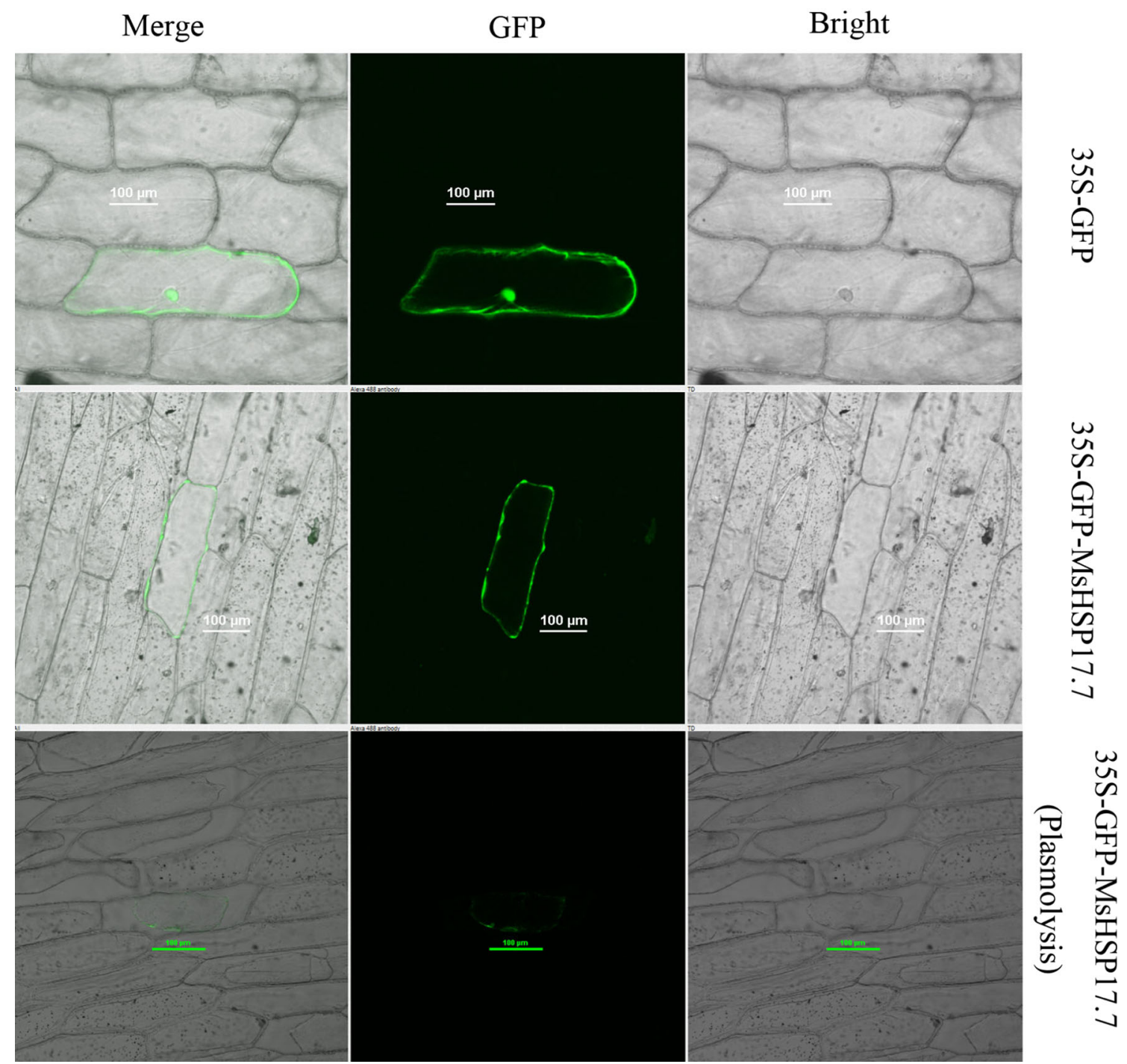

Fig. 3 Subcellular localization of the MsHSP17.7-GFP fusion in onion epidermal cells. GFP fluorescence was distributed throughout the entire cell in cells expressing the GFP empty vector. GFP fluorescence was localized to the cytoplasm of cells expressing the

MsHSP17.7 (GenBank accession: KJ621408). The gene encoded a 158 amino acid protein with a molecular weight of $17.67 \mathrm{kDa}$. The theoretical isoelectric point was 5.789 , which is characteristic of an acidic protein. The result from the ProtScale analysis indicated that most of the amino acids were hydrophilic (Supplementary Fig. 2A); therefore, MsHSP17.7 was deemed a hydrophilic protein. SignalP-4.1 identified no signal peptides in MsHSP17.7 (Supplementary Fig. 2B). In addition, the MsHSP17.7 protein was predicted to encode no transmembrane structures by TMHMM (Supplementary Fig. 2C). The protein secondary structure was predicted by Garnier [v6.0.1] to consist of $56.3 \% \alpha$-helix, $26.1 \% \quad \beta$-fold, $10.6 \% \quad \beta$-corner, and $18.3 \%$ random coil. Online software (ProtComp, http://
MsHSP17.7-GFP fusion protein. Plasmolysis of cells indicated MsHSP17.7-GFP fluorescence accumulated mainly in the cytoplasm instead of the cell wall. Bar $=100 \mu \mathrm{m}$

www.softberry.com) indicated that the MsHSP17.7 protein was likely to localize to the cytoplasm.

A multiple sequence alignment of the deduced MsHSP17.7 protein is shown in Fig. 1. MsHSP17.7 shares high protein sequence identity with MtHSP (93.98\%), PsHSP17.1 (83.13\%), GmHSP17.9 $(74.10 \%)$ and SlHSP17.6 (79.25\%), and it shares higher sequence similarity with dicotyledons compared with monocotyledons. Based on a comparison with cytosolic class II sHSP sequences, a unique domain (RDAKAMAATPADV) was found in the $\mathrm{N}$ terminus (Fig. 1). A conserved C-terminal domain ( $\alpha$-Crystallin domain, ACD) of approximately 90 amino acids contained consensus regions II and III. Additionally, a polyproline motif PPPEPKKP was identified at 


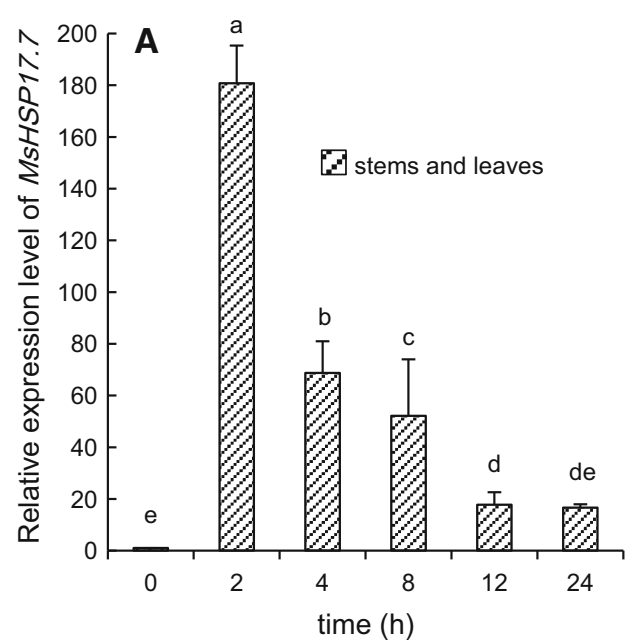

Fig. 4 RT-qPCR analysis of MsHSP17.7 in shoot (A) and root (B) tissues in response to heat shock $\left(37^{\circ} \mathrm{C}\right)$ treatments at different time intervals. After 0-24-h of heat treatment, the transcript abundance from 2-week-old alfalfa seedlings was determined.

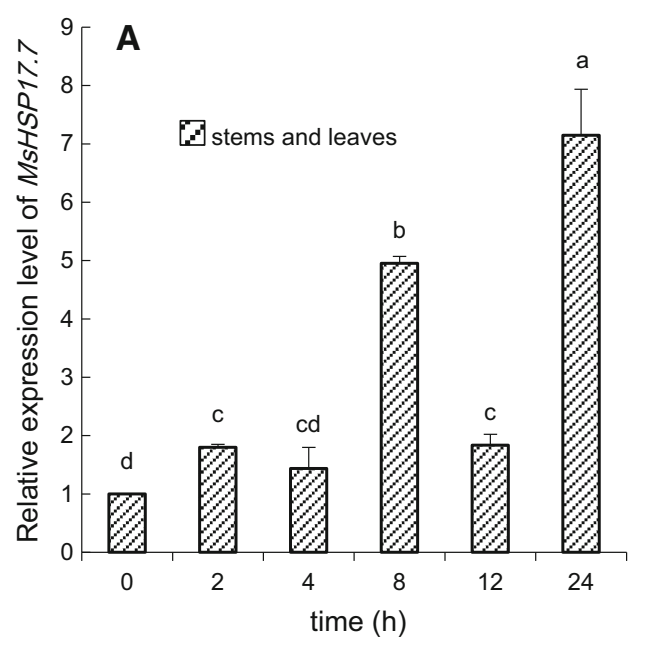

Fig. 5 RT-qPCR analysis of MsHSP17.7 in shoot (A) and root (B) tissues in response to $200 \mathrm{mM} \mathrm{NaCl}$ treatment at different time intervals. Transcript abundance from 2-week-old alfalfa seedlings was detected during a $24 \mathrm{~h}$ treatment. Vertical bars indicate the

the C-terminus [15-17]. A phylogenetic tree showed that MsHSP17.7 was identified as a member of the plant cytosolic class II sHSPs (Fig. 2).

\section{Subcellular localization of the MsHSP17.7}

GFP or the MsHSP17.7-GFP fusion protein was transiently expressed in onion epidermal cells. As shown in Fig. 3, the MsHSP17.7-GFP fusion protein accumulated mainly in the cytoplasm, whereas GFP alone was distributed throughout the entire cell. In addition to, the plasmolysis of onion cells indicated that MsHSP17.7-GFP fluorescence accumulated

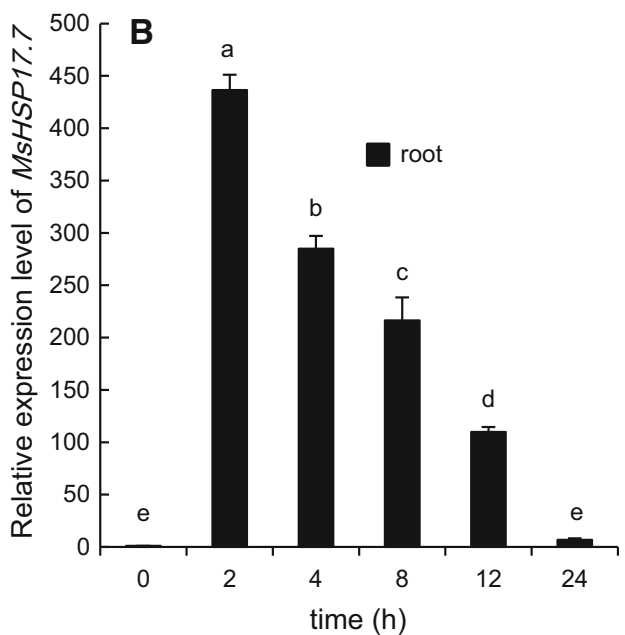

Vertical bars indicate the mean \pm SE of three biological independent experiments. The same letter is used to indicate no significant difference according to the LSD $t$ test $(P<0.05)$

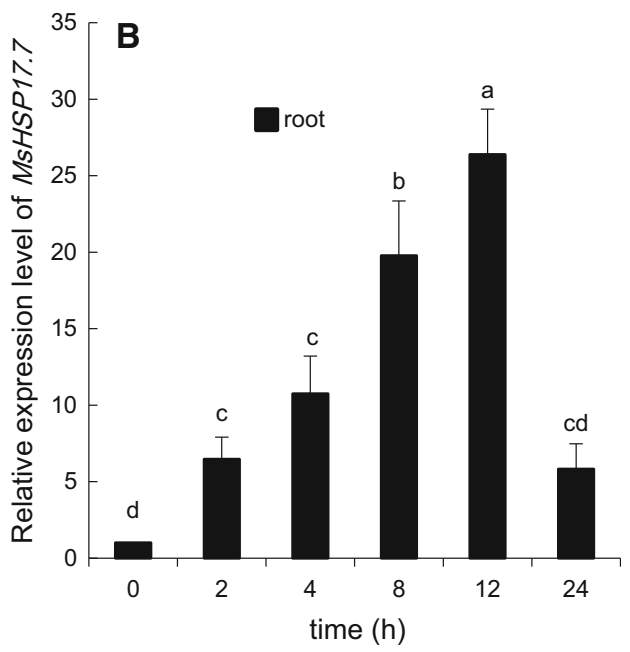

mean \pm SE of three biological independent experiments. The same letter is used to indicate no significant difference according to the LSD $t$ test $(P<0.05)$

mainly in the cytoplasm instead of cell wall. This result was consistent with the prediction that MsHSP17.7 by ProtComp online.

\section{Expression of MsHSP17.7 in alfalfa}

The relative expression levels of MsHSP17.7 mRNA under different stress conditions are shown in Figs. 4, 5, 6 and 7. Under heat shock stress (Fig. 4), the pattern of expression in stems and leaves was consistent with that in roots. After a rapid increase after $2 \mathrm{~h}$, the expression of mRNA MsHSP17.7 decreased as treatment time increased. At $2 \mathrm{~h}$, 
Fig. 6 RT-qPCR analysis of MsHSP17.7 in shoot (A) and root $(\mathbf{B})$ tissues in response to $15 \mathrm{mM} \mathrm{H} \mathrm{H}_{2} \mathrm{O}_{2}$ oxidative treatments at different time intervals. Transcript abundance from 2-week-old alfalfa seedlings was detected during a $24 \mathrm{~h}$ treatment. Vertical bars indicate the mean $\pm \mathrm{SE}$ of three biological independent experiments. The same letter is used to indicate no significant difference according to the LSD $t$ test $(P<0.05)$
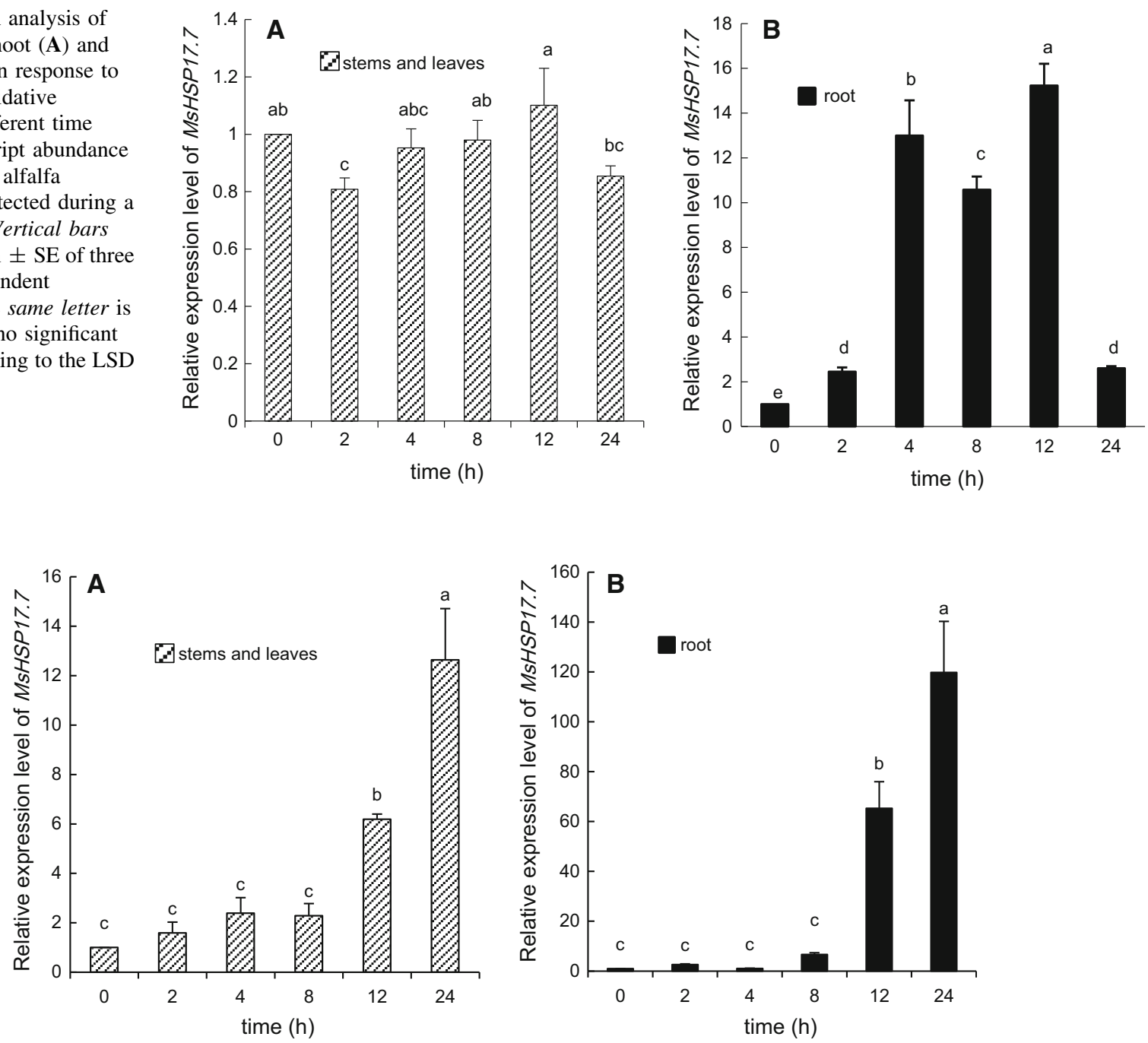

Fig. 7 RT-qPCR analysis of $M s H S P 17.7$ in shoot (A) and root (B) tissues in response to osmotic (200 $\mathrm{g}^{-1}$ PEG 6000) stress at different time intervals. Transcript abundance after a 0-24-h treatment of two-week-old alfalfa seedlings was detected. Vertical

the level of MsHSP17.7 mRNA in the above-ground parts was 180.7-fold higher than in the control, and the expression in the underground parts was 436.5-fold higher than in the control.

As shown in Fig. 5, the mRNA expression levels were higher under salt stress in the stems and leaves of stressed plants compared to those of control plants, except after $4 \mathrm{~h}$ of stress induction. However, there were no significant differences at 2, 4 and $12 \mathrm{~h}$. The mRNA expression level was 4-fold higher in the above-ground tissues of stressed plants than in those of the control plants at $8 \mathrm{~h}$. The expression level was the highest at $24 \mathrm{~h}$ (7-fold higher). The mRNA expression level of MsHSP17.7 in the root tissue gradually increased with treatment time until $12 \mathrm{~h}$, at which time the level was 25-times higher than in the roots of the control plants. The mRNA expression level in the bars indicate the mean $\pm \mathrm{SE}$ of three biological independent experiments. The same letter is used to indicate no significant difference according to the LSD $t$ test $(P<0.05)$

root suddenly decreased at $24 \mathrm{~h}$ of treatment to a level that was 5-fold higher than that of the control, whereas there was no obvious difference between expression levels at 0 and $24 \mathrm{~h}$ (Fig. 5B).

Upon peroxide stress treatment (Fig. 6A), the expression levels of MsHSP17.7 mRNA in the stems and leaves corresponded to those of the control at 4,8 and $12 \mathrm{~h}$. The expression levels at 2 and $24 \mathrm{~h}$ were slightly lower than those of the control at $0 \mathrm{~h}$. The MsHSP17.7 mRNA expression pattern in alfalfa root was completely different, increasing 1.5-, 12.0-, 9.5-, 14.2- and 1.6-fold at 2, 4, 8, 12, and $24 \mathrm{~h}$ (Fig. 6B).

The expression pattern of MsHSP17.7 in stems and leaves was consistent with that in roots under osmotic stress (Fig. 7A, B). MsHSP17.7 expression levels in the whole plant were not significantly different from those of the control from 0 to $8 \mathrm{~h}$. After $8 \mathrm{~h}$, the expression levels 


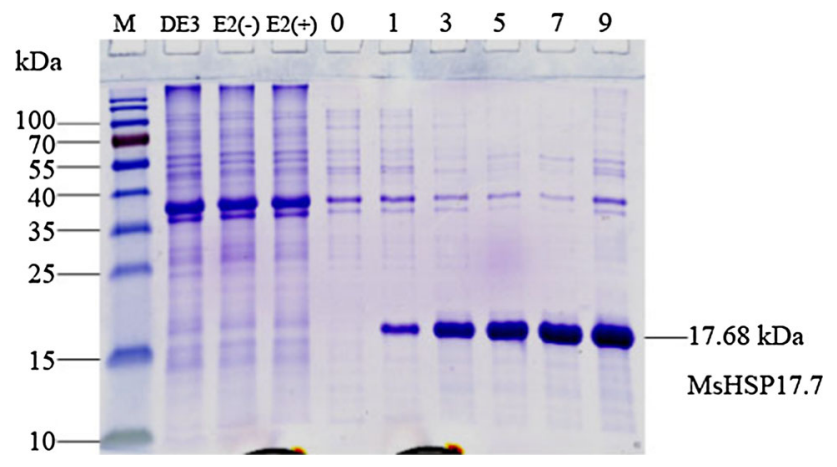

Fig. 8 Coomassie-stained gel was used in SDS-PAGE analysis of MsHSP17.7 fusion protein expression in E. coli. Lane $M$ protein ladder, lane DE3 E. coli strain DE3 total protein, lane E2 (-) pEASY-E2 total protein without ITPG, lane E2 (+) pEASY-E2 total protein with ITPG induction, lane 0 MsHSP17.7 soluble protein induction $0 \mathrm{~h}$ (+ITPG), lane 1, 3, 5, 7, 9 MsHSP17.7 soluble protein induction $1,3,5,7,9 \mathrm{~h}(+\mathrm{ITPG})$

increased. At $24 \mathrm{~h}$, the MsHSP17.7 mRNA expression level peaked at a 12-fold increase over controls in stems and leaves and at a 119-fold increase over controls in roots.

\section{Expression of MsHSP17.7 in E. coli and salinity and oxidative stress survival assays}

Under the $\mathrm{T} 7$ promoter, the MsHSP17.7 protein was abundantly expressed in E. coli (Fig. 8), and SDS-PAGE analysis clearly showed a $17.70 \mathrm{kDa}$ band. With the increased time of induction, the amount of IPTG-induced MsHSP17.7 expression was gradually increased (Fig. 8).

To evaluate whether MsHSP17.7 expression increased the capacity to resist salinity or peroxide stress in model microorganisms, the effects of MsHSP17.7 expression on the salinity and peroxide tolerance of $E$. coli were determined. In a controlled trial, as shown in Fig. 9A, there was no apparent difference in cell survival between the MsHSP17.7-expressing strain and the E2-expressing strain except at 2 and $4 \mathrm{~h}$. However, the MsHSP17.7-expressing strain showed increased $(P<0.05)$ survival after treatment with $200 \mathrm{mM} \mathrm{NaCl}$ compared to the vector control throughout the entire treatment period (Fig. 9B). Similarly, the MsHSP17.7-expressing strain showed tolerance to $15 \mathrm{mM} \mathrm{H}_{2} \mathrm{O}_{2}$ treatment (Fig. 9C).
Fig. 9 The effects of MsHSP17.7 expression on the growth of E. coli under salt and peroxide stress conditions. Compared to the control of E. coli in normal condition (A), E. coli cell growth after $100 \mathrm{mM} \mathrm{NaCl}$ treatment (B) and $15 \mathrm{mM} \mathrm{H}_{2} \mathrm{O}_{2}$ treatment (C) was determined. E2 denoted E. coli with pEASY-E2 and represented control in (A-C). MsHSP17.7 denoted E. coli with MsHSP17.7. The mean value of each transgenic line represented a statistically significant difference with respect to the control, as determined by the LSD $t$ test $(P<0.05)$. Vertical bars indicate the mean $\pm \mathrm{SE}$ of three biological independent experiments
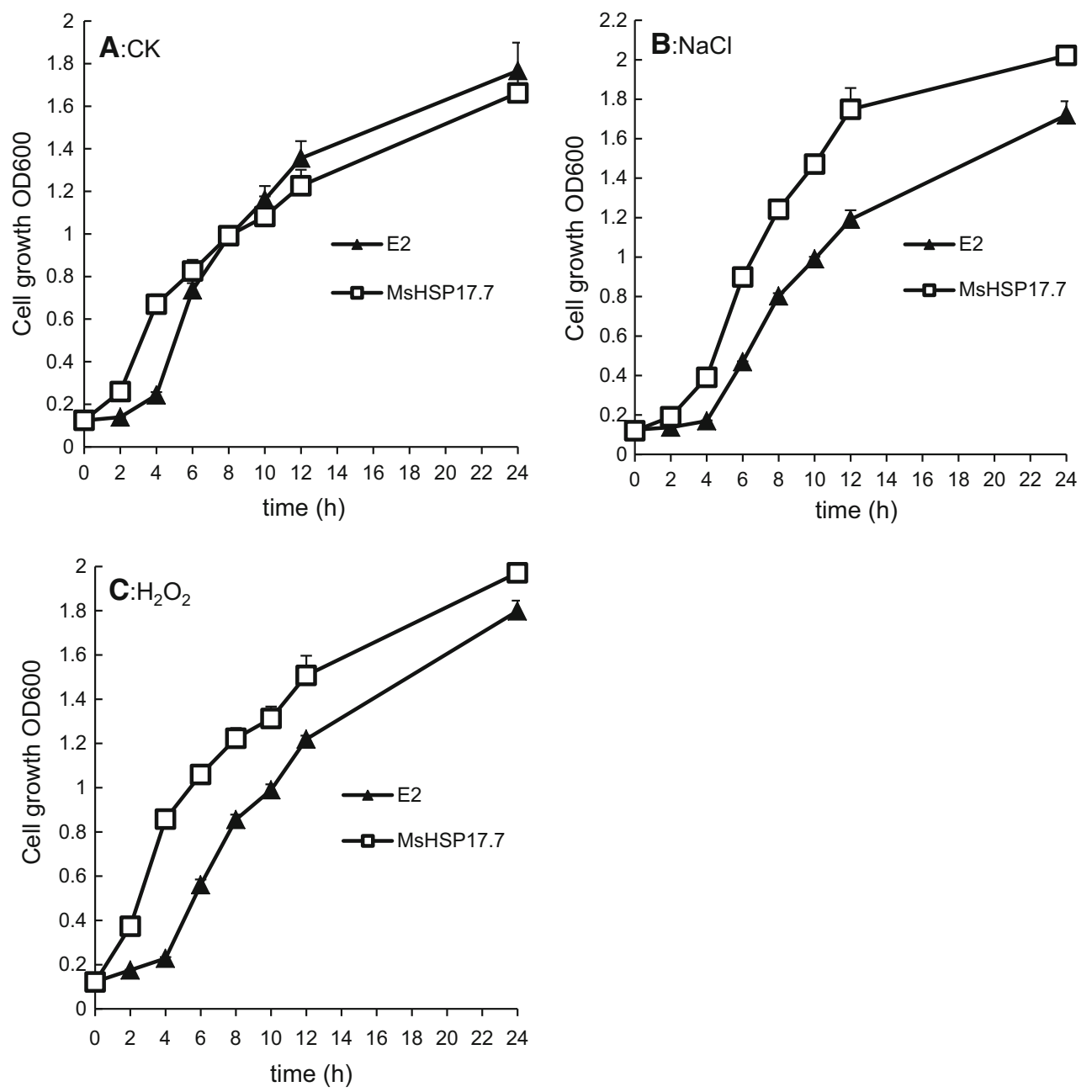


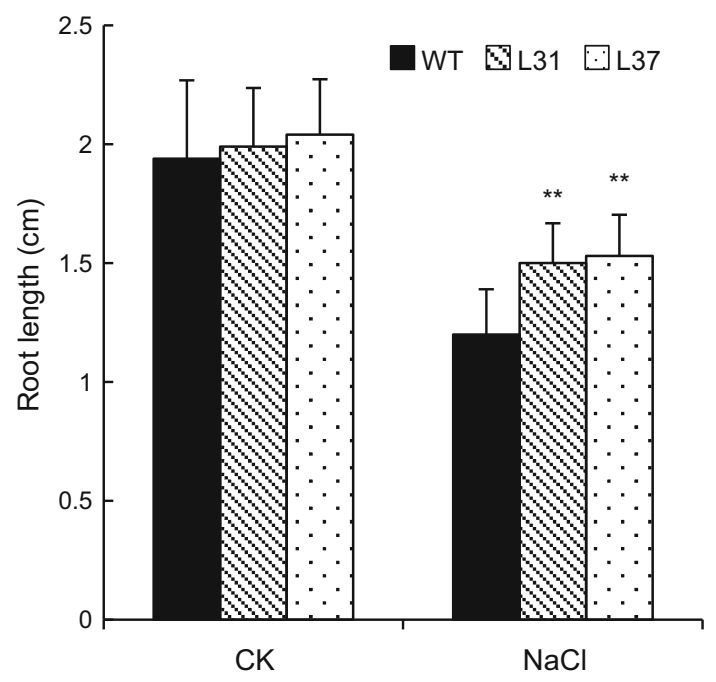

Fig. 10 Root length of transgenic and wild-type Arabidopsis under salt stress $(150 \mathrm{mM} \mathrm{NaCl})$. Double asterisk indicates highly significant difference compared with wild-type Arabidopsis according to the LSD $t$ test $(P<0.01)$. Vertical bars indicate the mean \pm SE of three independent experiments

\section{Analysis of transgenic Arabidopsis under stress conditions}

In the transgenic lines L31 and L37 under normal growth conditions, the root lengths were 1.99 and $2.04 \mathrm{~cm}$, respectively, as shown in Fig. 10, which were not significantly different from the wild-type line. Under salt stress, the root lengths of transgenic Arabidopsis lines were 1.5 and $1.53 \mathrm{~cm}$, which were 0.3 and $0.33 \mathrm{~cm}$ longer than the wild-type line $(P<0.01)$.

Under control conditions, the MsHSP17.7-overexpressing lines did not show significant differences in proline or

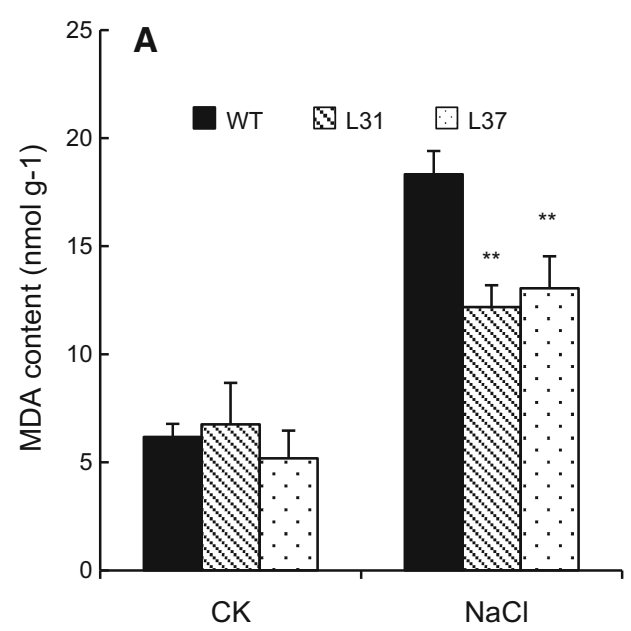

Fig. 11 MDA (A) and proline (B) contents were measured in transgenic and wild-type Arabidopsis under salt stress condition $(200 \mathrm{mM} \mathrm{NaCl})$. Double asterisk indicates highly significant
MDA levels compared with wild-type Arabidopsis (Fig. 11). However, after treatment with $200 \mathrm{mM} \mathrm{NaCl}$, the MDA content of the L31 and L37 lines were 12.2 and $13.0 \mathrm{nmol} \mathrm{g}^{-1}$, respectively, and were significantly lower that of the wild-type line (Fig. 11A). There was no significant difference in the proline level between the transgenic and wild-type lines (Fig. 11B).

\section{Discussion}

Strategies employed by higher plants for surviving adverse conditions include behavioral adaptations, morphological changes and physiological regulation [18]. The role of sHSPs in protecting cells against damage related to abiotic and biotic stresses has been well demonstrated in organisms ranging from fungi $[19,20]$ to plants $[16,21]$. The functions of sHSPs include binding to unfolded proteins and regulating their intracellular distribution, protein degradation and signal transduction, allowing cell survival under stress conditions [9]. In spite of the considerable research on the role of $s H S P$ in response to environmental stresses in diverse plants, few studies on alfalfa $s H S P$ have been reported. We cloned and characterized the MsHSP17.7 gene from alfalfa. A phylogenetic tree analysis divided 50 sHSPs into six families, of which Classes CI, CII and CIII are localized in the cytosol or nucleus [4]. Additionally, subcellular localization studies demonstrated that MsHSP17.7 is located in the cytosol. Combined with the evolutionary tree analysis, we propose that MsHSP17.7 is a cytosolic Class II sHSP.

sHSP genes are highly induced under conditions ranging from abiotic exposure to biotic stresses. It has been reported that A. thaliana CI sHSPs (Hsp17.4CI,

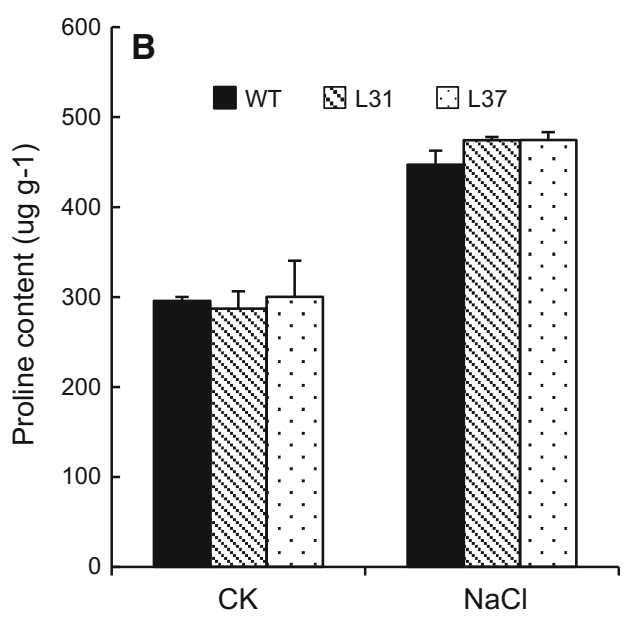

difference compared with wild-type Arabidopsis according to the LSD $t$ test $(P<0.01)$. Vertical bars indicate the mean \pm SE of three independent experiments 
Hsp17.6ACI, Hsp17.6BCI, and Hsp17.6CCI) are expressed during heat stress, osmotic stress, oxidative stress, UV-B exposure and other abiotic stressors [9]. HSPs are induced by heat, cold, drought, and oxidative and salt stresses in Oryza sativa [22]. In addition, Neta-Sharir demonstrated that tomato chloroplast $s H S P 21$ is induced by heat treatment in leaves [23].

In this paper, the abundantly increased MsHSP17.7 mRNA indicated adaptation of the plant to the adverse environments. sHSP genes also respond to osmotic and salt stress. Ruibal et al. [24] reported that PpHsp16.4 was upregulated after exposure to various abiotic stress factors, including strong light, heat, salt and osmotic stress. In this study, MsHSP17.7 mRNA was up-regulated by $\mathrm{NaCl}$ and PEG treatments, suggesting that MsHSP17.7 might play a key role in osmotic and salt stress. AtHSP15.7, a peroxisomal $s H S P$, has been shown to be strongly induced by both oxidative and heat stress [11]. However, the analysis of an Arabidopsis cytosolic class II sHSP, AtHSP17.6A, revealed that this $S H S P$ was induced by osmotic but not oxidative stress [25]. In our study, MsHSP17.7 mRNA expression was regulated in alfalfa roots under oxidative stress, but its levels remained stable in the stems and leaves. The At-HSP17.6A protein was not detected in PEGtreated Arabidopsis plants, whereas At-HSP17.6A mRNA was induced [15]. In our study, MsHSP17.7 was induced after chronic drought treatment. Therefore, MsHSP17.7 was presumed to be involved in the drought response and to play a crucial role in plant stress tolerance. Under high temperature, drought, high salt concentration, or exposure to various pathogens [26], the primary function of this sHSP is to promote refolding of non-native proteins that have been denatured under stress condition.

Previous studies have demonstrated that the constitutive overexpression of sHSPs in plants is associated with enhanced resistance to abiotic stress $[15,16]$. In this study, MsHSP17.7-expressing E. coli showed increased survival following salt stress and peroxide stress, respectively, compared with controls. In Arabidopsis, overexpression of wheat chloroplastic sHSP26 results in improved heat tolerance [27]. In this study, the transgenic Arabidopsis seedlings exhibited significantly longer root, lower MDA content and similar proline content compared to wild-type Arabidopsis under high salinity stress. The lower MDA content indicated that less damage occurred in the plant. Some researchers argue that proline is a compatible osmolyte that accumulates in plant cells in response to salt stress [28], but others favor the view that proline is simply a stress-induced product [29]. In the present study, there was no marked difference in the proline content between transgenic and wild-type Arabidopsis.

In conclusion, we showed that MsHSP17.7 is localized in the cytoplasm and is induced by heat shock, high salinity, peroxide and drought stress. Expression of MsHSP17.7 in transgenic E. coli and A. thaliana indicated that it could enhance salt tolerance.

Acknowledgments This work was supported by the National Program on Key Basic Research Project (grant numbers: 2014CB138703-2), the China Agriculture Research System (CARS35-04) and the Agricultural Science and Technology Innovation Program (ASTIP-IAS14).

Open Access This article is distributed under the terms of the Creative Commons Attribution 4.0 International License (http://crea tivecommons.org/licenses/by/4.0/), which permits unrestricted use, distribution, and reproduction in any medium, provided you give appropriate credit to the original author(s) and the source, provide a link to the Creative Commons license, and indicate if changes were made.

\section{References}

1. Wang W, Vinocur B, Shoseyov O, Altman A (2004) Role of plant heat-shock proteins and molecular chaperones in the abiotic stress response. Trends Plant Sci 9(5):244-252

2. Timperio AM, Egidi MG, Zolla L (2008) Proteomics applied on plant abiotic stresses: role of heat shock proteins (HSP). J Proteomics 71(4):391-411

3. Lee KW, Cha JY, Kim KH, Kim YG, Lee BH, Lee SH (2012) Overexpression of alfalfa mitochondrial HSP23 in prokaryotic and eukaryotic model systems confers enhanced tolerance to salinity and arsenic stress. Biotechnol Lett 34(1):167-174

4. Scharf KD, Siddique M, Vierling E (2001) The expanding family of Arabidopsis thaliana small heat stress proteins and a new family of proteins containing alpha-crystallin. Cell Stress Chaperones 6(3):225-237

5. Waters ER, Lee GJ, Vierling E (1996) Evolution structure and function of the small heat shock protein in plants. J Exp Bot 47:325-338

6. Sato Y, Yokoya S (2008) Enhanced tolerance to drought stress in transgenic rice plants overexpressing a small heat-shock protein, sHSP17.7. Plant Cell Rep 27(2):329-334

7. Nakamoto L, Vigh L (2007) The small heat shock proteins and their clients. Cell Mol Life Sci 64:294-306

8. Hilton GR, Lioe H, Stengel F, Baldwin AJ, Benesch JL (2013) Small heat-shock proteins: paramedics of the cell. Top Curr Chem 328:69-98

9. Waters ER (2013) The evolution, function, structure, and expression of the plant sHSPs. J Exp Bot 64(2):391-403

10. Song L, Jiang Y, Zhao H, Hou M (2012) Acquired thermotolerance in plants. Plant Cell Tissue Organ Cult 111(3):265-276

11. Ma C, Haslbeck M, Babujee L, Jahn O, Reumann S (2006) Identification and characterization of a stress-inducible and a constitutive small heat-shock protein targeted to the matrix of plant peroxisomes. Plant Physiol 141(1):47-60

12. Tamura K, Peterson D, Peterson D, Stecher G, Nei M, Kumar S (2011) MEGA5: molecular evolutionary genetics analysis using maximum likelihood, evolutionary distance, and maximum parsimony method. Mol Biol Evol 28:2731-2739

13. Clough SJ, Bent AF (1998) Floral dip: a simplified method for Agrobacterium-mediated transformation of Arabidopsis thaliana. Plant J 16:735-743

14. Liu YG, Chen Y, Zhang Q (2005) Amplification of genomic sequences flanking T-DNA insertions by thermal asymmetric 
interlaced polymerase chain reaction. Methods Mol Biol 286:341-348

15. Xue Y, Peng R, Xiong A, Li X, Zha D, Yao Q (2010) Overexpression of heat shock protein gene hsp26 in Arabidopsis thaliana enhances heat tolerance. Biol Plant 54(1):105-111

16. Zhou Y, Chen H, Chu P, Li Y, Tan B, Ding Y, Tsang EW, Jiang L, Wu K, Huang S (2012) NnHSP17.5, a cytosolic class II small heat shock protein gene from Nelumbo nucifera, contributes to seed germination vigor and seedling thermotolerance in transgenic Arabidopsis. Plant Cell Rep 31(2):379-389

17. Bondino HG, Valle EM, Ten Have A (2012) Evolution and functional diversification of the small heat shock protein/alphacrystallin family in higher plants. Planta 235(6):1299-1313

18. Fu W, Yao J, Wang X, Liu F, Fu G, Duan D (2009) Molecular Cloning and Expression Analysis of a Cytosolic Hsp70 gene from Laminaria japonica (Laminariaceae, Phaeophyta). Mar Biotechnol 11(6):738-747

19. Mayer FL, Wilson D, Jacobsen ID, Miramon P, Slesiona S, Bohovych IM, Brown AJ, Hube B (2012) Small but crucial: the novel small heat shock protein Hsp21 mediates stress adaptation and virulencein Candida albicans. PLoS One 7:e38584

20. Anupama G (2014) Small heat shock proteins (HSP12, HSP20 and HSP30) play a role in Ustilago maydis pathogenesis. FEMS Microbiol Lett 361:17-24

21. Zhang L, Gao Y, Pan H, Hu W, Zhang Q (2013) Cloning and characterisation of a Primula heat shock protein gene, PfHSP17.1, which confers heat, salt and drought tolerance in transgenic Arabidopsis thaliana. Acta Physiologiae Plant 35(11):3191-3200

22. Hu WH, Hu GC, Han B (2009) Genome-wide survey and expression profiling of heat shock proteins and heat shock factors revealed overlapped and stress specific response under abiotic stresses in rice. Plant Sci 176(4):583-590

23. Neta-Sharir I, Isaacson T, Lurie S, Weiss D (2005) Dual role for tomato heat shock protein 21: protecting photosystem II from oxidative stress and promoting color changes during fruit maturation. Plant Cell 17(6):1829-1838

24. Ruibal C, Castro A, Carballo V, Szabados L, Vidal S (2013) Recovery from heat, salt and osmotic stress in Physcomitrella patens requires a functional small heat shock protein PpHsp16.4. BMC Plant Biol 13:174

25. Sun W, Bernard C, Cotte B, Van Montagu M, Verbruggen N (2001) At-HSP17.6A, encoding a small heat-shock protein in Arabidopsis, can enhance osmotolerance upon overexpression. Plant J 27(5):407-415

26. Liu HY, Dicksved J, Lundh T, Lindberg JE (2014) Expression of heat shock protein 27 and 72 correlates with specific commensal microbes in different regions of porcine gastrointestinal tract. Am J Physiol Gastrointest Liver Physiol 306:1033-1041

27. Chauhan H, Khurana N, Nijhavan A, Khurana JP, Khurana P (2012) The wheat chloroplastic small heat shock protein (sHSP26) is involved in seed maturation and germination and imparts tolerance to heat stress. Plant Cell Environ 35(11):1912-1931

28. Aida HS, Radhia GB, Bidani A, Jaoua L, Samir J (2005) Overexpression of 1-pyrroline-5-carboxylate synthetase increases proline production and confers salt tolerance in transgenic potato plants. Plant Sci 169(4):746-752

29. Liu JP, Zhu JK (1997) Proline accumulation and salt-stress-induced gene expression in a salt-hypersensi-tivemutant of Arabidopsis. Plant Physiol 114:591-596 